\title{
ORIGINAL ARTICLE \\ Prognostic factors for cervical spondylotic amyotrophy: are signs of spinal cord involvement associated with the neurological prognosis?
}

\author{
Y Iizuka, H Iizuka, T Mieda, R Kobayashi, S Tsutsumi, T Nakajima, Y Sorimachi, T Ara, M Nishinome, \\ T Seki and K Takagishi
}

\begin{abstract}
Objectives: The purpose of this study was to clarify the prognostic factors for cervical spondylotic amyotrophy (CSA).
Methods: The authors retrospectively reviewed the medical records of 47 consecutive patients with CSA in whom the presence/ absence of the pyramidal tract sign was noted. We analyzed whether the age, sex, presence of diabetes mellitus, medication (vitamin B12), type of the most atrophic and impaired muscle, the muscle strength at the presentation, the presence of the pyramidal tract sign, magnetic resonance imaging (MRI) findings, including the presence and number of T2 high signal intensity areas (T2 HIA) in the spinal cord and the conversion to surgery were associated with the recovery of muscle strength in the patients. In addition, we also investigated whether the duration of symptoms before surgery and the type of surgery were associated with the recovery of muscle strength in patients who required conversion to surgical treatment.

Results: The presence of $\mathrm{T} 2 \mathrm{HIA}$ on MRI $(P=0.002)$, the number of T2 HIA on MRI $(P=0.002)$ and conversion to surgery $(P=0.015)$ were found to be significantly associated with a poorer recovery at the observational final follow-up. Further, the presence of the pyramidal tract sign $(P=0.043)$ was significantly associated with a poor recovery at the final follow-up after surgery.

Conclusion: The presence of a high signal intensity change on T2-weighted MRI and the pyramidal tract sign can be used as prognostic factors for patients with CSA.
\end{abstract}

Spinal Cord (2014) 52, 364-367; doi:10.1038/sc.2014.23; published online 11 March 2014

Keywords: cervical spondylotic amyotrophy; magnetic resonance imaging; increased signal intensity; pyramidal tract sign; prognostic factor

\section{INTRODUCTION}

Cervical spondylosis is a degenerative change of the cervical spine, and it has been known that it sometimes induces neurological symptoms as a result of spinal cord and/or nerve root compression induced by the degenerative changes of the cervical spine.

In 1952, Brain et al. ${ }^{1}$ first described cervical spondylosis cases that had muscle atrophy in the upper extremities without sensory disturbance or pyramidal tract signs. In 1965, Keegan ${ }^{2}$ reported a case of cervical spondylosis with dissociated motor loss in the upper extremity, which was characterized by severe atrophy of the deltoid and biceps brachii muscles, mild sensory disturbance, no pyramidal tract sign and selective compression on the anterior nerve root of the cervical spine demonstrated by a pathological examination. Furthermore, the clinical entity of cervical spondylotic amyotrophy (CSA) was established by Sobue et al. ${ }^{3}$ in 1975 , and they indicated that its main clinical symptoms are muscular atrophy or weakness of the upper extremities with no or insignificant sensory disturbance and the presence of the pyramidal tract sign reflecting the involvement of the white matter of the spinal cord in lower extremities was an acceptable finding. ${ }^{3,4}$

However, in the previous studies, whether or not the authors accepted the presence of the pyramidal tract sign varied, as its inclusion in the diagnostic criteria for CSA has been controversial, or was obscure in some of the previous studies. The purpose of this study was to investigate several factors in CSA patients who were diagnosed, accepting the presence of the pyramidal tract sign, and to clarify whether or not these factors, including the magnetic resonance imaging (MRI) findings and the presence of the pyramidal tract sign are associated with the neurological prognosis.

\section{MATERIALS AND METHODS}

We retrospectively reviewed the medical records of 47 consecutive patients who were diagnosed with CSA between January 2003 and December 2012 at our University Hospital. The average patient age at the onset of the symptoms was 61.5 years (range, 37-82 years). There were 38 men and 9 women. The average duration from the onset of symptoms to the final follow-up was 30.0 months (range, 6-192 months). We included the patients with CSA in this study when the following criteria were fulfilled: (1) degenerative changes identified by $\mathrm{X}$-ray of the cervical spine, (2) unilateral muscular weakness scored between 0 and 3 on the manual muscle test (MMT) of any muscle of the upper extremities, (3) no or insignificant sensory disturbance and (4) the presence or absence of the pyramidal tract sign in the lower extremities.

Patients who had a past history of cervical spine surgery, ossification of the posterior longitudinal ligament of the cervical spine or a diagnosis of motor neuron diseases ascertained by a neurologist were excluded from this study. Moreover, we excluded CSA patients who had muscular atrophy or weakness of the bilateral upper extremities to avoid any confusion. ${ }^{5}$ 
We retrospectively collected data from these patients with CSA regarding the age, sex, presence of diabetes mellitus, medication (vitamin B12), type of the most atrophic and impaired muscle (proximal type: impairment of the deltoid and biceps brachii muscles, distal type: impairment of the triceps, forearm and hand intrinsic muscles), MMT score of the muscles in the upper extremities at the time of presentation as determined by at least two authors, the presence of the pyramidal tract sign, the presence and number of T2 high signal intensity areas (T2 HIA) in the spinal cord were evaluated using axial and sagittal MRI by two authors (YI and HI), and the need for conversion to surgery after the observational follow-up. Moreover, we also collected data regarding the duration of symptoms before surgery and the type of surgery in patients who required conversion to surgical treatment.

In order to evaluate the recovery of the muscle strength of the most atrophic and impaired muscle, we used the MMT and classified patients into four recovery grades: 'excellent', full recovery or recovery to a MMT grade of 2; 'good', one grade of recovery; 'fair', no improvement and 'poor', worsening of the condition. Furthermore, we categorized patients into two groups based on the results of the recovery grade of the muscle strength, that is, a good recovery group including patients with 'excellent' and 'good' outcomes and a poor recovery group including patients with 'fair' and 'poor' outcomes.

We investigated whether any of these factors had an impact on the neurological prognosis of CSA using a statistical analysis.

\section{Statistical analysis}

The data were expressed as mean \pm s.d. The statistical analysis was carried out using Fisher's exact probability test, the $\chi^{2}$-test and Mann-Whitney's $U$-test. We used a Spearman's correlation coefficient analysis to determine the correlations between variables. All of the analyses were conducted using the IBM SPSS Statistics 19 software program (IBM Japan, Tokyo, Japan), and a value of $P<0.05$ was considered to be significant.

\section{RESULTS}

Evaluation of the recovery of muscle strength at the final observational follow-up

For all 47 patients with CSA, an observational follow-up examination was performed as the initial management. The duration from the onset of symptoms to recovery assessment at the final observational follow-up was $18.0 \pm 33.7$ months. The characteristics of the 47 patients are summarized in Table 1 . Of these 47 patients, 16 patients were classified as having an excellent outcome, 6 as good, 24 as fair and one as poor, respectively, at the final observational follow-up. Therefore, 22 patients were categorized as the good recovery group and 25 as the poor recovery group. There were no significant differences between the two groups regarding the age, sex, diabetes mellitus, medication (vitamin B12), type of the most atrophic and impaired muscle, MMT score of muscles at the presentation or presence/absence of the pyramidal tract sign. On the other hand, the presence of T2 HIA on MRI $(P=0.002)$ and the number of T2 HIA on MRI $(P=0.002)$ and the number of patients requiring conversion to surgery $(P=0.015)$ were found to be significantly higher in the poor recovery group compared with the good recovery group (Table 2). Furthermore, the presence of T2 HIA on MRI was significantly associated with the need for conversion to surgery $(P=0.036)$, and the recovery grade of the muscle strength had a significant negative correlation with the number of T2 HIA on MRI $(r=-0.479, P=0.001)$.

Evaluation of the recovery of muscle strength at the final follow-up after surgery

After the initial observational follow-up, 18 of the 47 patients underwent surgical treatment, including 4 cases of anterior discectomy and fusion and 14 cases of laminoplasty via the posterior approach. The duration from the onset of symptoms to recovery
Table 1 Demographic and clinical characteristics of 47 patients with CSA

\begin{tabular}{lc} 
Factor & Value \\
\hline Age (years) & $61.5 \pm 10.0$ \\
Sex (M/F) & $38 / 9$ \\
DM & 4 \\
Vitamin B12 & 19
\end{tabular}

$\begin{array}{lc}\text { Type of impaired muscle } & \\ \text { Proximal type } & 35 \\ \text { Distal type } & 12 \\ \text { MMT at the presentation } & 1.7 \pm 0.9 \\ \text { Pyramidal tract sign } & 18 \\ \text { Presence of T2 HIA } & 15 \\ \text { Numbers of T2 HIA } & 0.3 \pm 0.6 \\ & \\ \text { Recovery grade } & 16 \\ \text { Excellent } & 6 \\ \text { Good } & 24 \\ \text { Fair } & 1 \\ \text { Poor } & 18 \\ \text { Conversion to surgery } & \end{array}$

Abbreviations: CSA, cervical spondylotic amyotrophy; DM, diabetes mellitus; F, female; M, male; MMT, manual muscle test; T2 HIA, T2 high signal intensity areas.

Table 2 Factor comparison for 47 patients with CSA stratified by recovery status

\begin{tabular}{lccc}
\hline Factor & Good $(\mathrm{n}=22)$ & Poor $(\mathrm{n}=25)$ & P-value \\
\hline Age (years) & $60.1 \pm 11.5$ & $62.8 \pm 8.7$ & $0.38^{\mathrm{a}}$ \\
Sex (M/F) & $17 / 5$ & $21 / 4$ & $0.71^{\mathrm{b}}$ \\
DM & 2 & 2 & $1.00^{\mathrm{b}}$ \\
Vitamin B12 & 10 & 9 & $0.56^{\mathrm{b}}$ \\
& & & \\
Type of impaired muscle & 19 & 16 & $0.10^{\mathrm{b}}$ \\
$\quad$ Proximal type & 3 & 9 & \\
$\quad$ Distal type & $1.9 \pm 0.9$ & $1.6 \pm 0.8$ & $0.22^{\mathrm{a}}$ \\
MMT at the presentation & 6 & 12 & $0.14^{\mathrm{c}}$ \\
Pyramidal tract sign & 2 & 13 & $0.002^{\mathrm{b}}$ \\
Presence of T2 HIA & 0.09 & 0.64 & $0.002^{\mathrm{a}}$ \\
Numbers of T2 HIA & 4 & 14 & $0.01^{\mathrm{b}}$ \\
Conversion to surgery & & & \\
\hline
\end{tabular}

Abbreviations: CSA, cervical spondylotic amyotrophy; DM, diabetes mellitus; $F$, female; $M$, male; MMT, manual muscle test; T2 HIA, T2 high signal intensity areas. aann-Whitney U-test.

b $\chi^{2}$-test.

cFisher's exact probability test.

assessment at the final follow-up after surgery was $40.4 \pm 26.1$ months. The characteristics of these 18 patients are provided in Table 3. Of these 18 patients, 10 patients were classified as excellent, 5 as good, 2 as fair and 1 as poor, respectively, at the final follow-up after surgery. Therefore, 15 patients were categorized in the good recovery group and three in the poor recovery group. There were no significant differences between the two groups regarding the age, sex, duration of symptoms before surgery, diabetes mellitus, type of the most atrophic and impaired muscle, MMT score of muscles at the presentation, the presence and number of T2 HIA on sagittal MRI and the type of surgery. However, the presence of the pyramidal tract sign $(P=0.043)$ was significantly associated with a poor recovery at the final follow-up after surgery (Table 4 ). 
Table 3 Demographic and clinical characteristics of 18 patients of conversion to surgery

\begin{tabular}{lc}
\hline Factor & Value \\
\hline Age (years) & $58.9 \pm 10.8$ \\
Sex (M/F) & $16 / 2$ \\
DM & 2 \\
& \\
Type of impaired muscle & 13 \\
Proximal type & 5 \\
Distal type & $1.5 \pm 0.9$ \\
MMT at the presentation & 7 \\
Pyramidal tract sign & 9 \\
Presence of T2 HIA & $0.5 \pm 0.6$ \\
Numbers of T2 HIA & 8.7 \\
Duration before surgery (months) & \\
Type of surgery & \\
ADF & \\
Laminoplasty & 4 \\
Recovery grade & 14 \\
Excellent & \\
Good & \\
Fair & \\
Poor & \\
\hline Abbrevions: ADF, anteris discetong & \\
\hline
\end{tabular}

Abbreviations: ADF, anterior discectomy and fusion; DM, diabetes mellitus; F, female; M, male; MMT, manual muscle test; T2 HIA, T2 high signal intensity areas.

Table 4 Factor comparison for 18 patients of conversion to surgery stratified by recovery status

\begin{tabular}{lccc}
\hline Factor & Good $(\mathrm{n}=15)$ & Poor $(\mathrm{n}=3)$ & P-value \\
\hline Age (years) & $59.5 \pm 11.7$ & $56.0 \pm 4.5$ & $0.40^{\mathrm{a}}$ \\
Sex (M/F) & $14 / 1$ & $2 / 1$ & $0.31^{\mathrm{b}}$ \\
DM & 2 & 0 & $1.00^{\mathrm{b}}$ \\
& & & \\
Type of impaired muscle & 10 & 3 & $0.52^{\mathrm{b}}$ \\
$\quad$ Proximal type & 5 & 0 & \\
$\quad$ Distal type & $1.4 \pm 0.9$ & $2.0 \pm 1.0$ & $0.40^{\mathrm{a}}$ \\
MMT at the presentation & 4 & 3 & $0.04^{\mathrm{b}}$ \\
Pyramidal tract sign & 7 & 2 & $1.00^{\mathrm{b}}$ \\
Presence of T2 HIA & $0.4 \pm 0.5$ & $1.0 \pm 1.0$ & $0.28^{\mathrm{a}}$ \\
Numbers of T2 HIA & 6.9 & 18.0 & $0.21^{\mathrm{a}}$ \\
Duration before surgery (months) & & & \\
& & & \\
Type of surgery & 4 & 0 & $1.00^{\mathrm{b}}$ \\
$\quad$ ADF & 11 & 3 & \\
Laminoplasty & & &
\end{tabular}

Abbreviations: ADF, anterior discectomy and fusion; DM, diabetes mellitus; F, female; M, male; MMT, manual muscle test; T2 HIA, T2 high signal intensity areas.

a Mann-Whitney U-test.

${ }^{\mathrm{b}}$ Fisher's exact probability test.

\section{DISCUSSION}

Concerning the pathophysiology of CSA, it has been speculated that the damage to the gray matter, including the anterior horn of the spinal cord or anterior nerve root, induces symptoms including muscular atrophy or weakness of the upper extremities in patients with CSA. It has also been reported that vascular insufficiency to the anterior horn may be associated with the onset of the symtoms. ${ }^{2,4,6-8}$
Furthermore, it has been reported that neurological symptoms of the gray matter of the spinal cord occur as a result of spinal cord compression by the cervical degenerative changes in patients with the early stage of cervical spondylotic myelopathy (CSM), and later, the compressive spinal cord lesion in CSM gradually spreads to the white matter. ${ }^{9}$ Therefore, CSA has been considered to be a subtype of CSM, that is, the selective damage to the anterior horn in the gray matter induced by the cervical degenerative changes in CSA resembles the early stage CSM. However, whether or not the presence of the pyramidal tract sign reflecting the involvement of the white matter is accepted in the inclusion criteria for CSA is controversial, ${ }^{2,3,10}$ and it has also not been clearly described in many of the previous studies. In this study, we included patients with CSA in whom the presence of pyramidal tract sign was evaluated and accepted in the inclusion criteria, and investigated the prognostic factors for the pathology in these patients.

In this study, we found that the pyramidal tract sign reflecting white matter involvement was significantly associated with the neurological prognosis in CSA patients after surgical treatment. Therefore, based on our results, it was considered that the presence of the pyramidal tract sign can be used as a poor prognostic factor in surgically treated CSA patients. However, Inui et al. ${ }^{11}$ reported that the pyramidal tract sign was not associated with the prognosis, not only in conservatively treated patients, but also in surgically treated patients. Although further investigations will be needed to explain the inconsistencies between the studies, the differences in the regimen used for conservative management may be at least partly responsible. That is, the use of preoperative continuous cervical traction for CSA patients for at least two weeks in their study might have affected the prognosis of surgically treated patients who were positive for the pyramidal tract sign after conservative management, whereas no special intervention was applied as initial observational management for most of the CSA patients in this study. In addition, some patients received vitamin B12; however, such administration had no significant impact on the neurological prognosis.

There have been several reports regarding the association between CSM and the MRI findings of the cervical spine, including the signal intensity change of the spinal cord on T2-weighted images. It has been reported that those signal intensity changes are associated with a poor neurological prognosis after decompression surgery for CSM. ${ }^{12-15}$ On the other hand, Tauchi et al. ${ }^{16}$ recently reported that the MRI findings of the spinal cord were not associated with the surgical outcome in CSA patients, and they speculated that the compression or damage in the cervical spine was localized in the anterior horn of the spinal cord or the ventral nerve root in affected CSA patients, although it exists in the central portion of the spinal cord in most CSM patients. In the present study, we investigated the association between the signal intensity changes of the spinal cord on T2-weighted MR images and the neurological prognosis not only at the final observational follow-up, but also at the final follow-up after conversion to surgery, and we showed that CSA patients with high signal intensity changes on T2-weighted MRI had a poorer neurological prognosis compared with those without the high signal intensity change, although no significant difference was noted when the analysis was limited to the patients who required conversion to surgery. On the basis of these results, we considered that the presence and number of the high signal intensity areas on T2-weighted MRI can be used as a prognostic factor to predict the neurological prognosis in observational follow-up patients, and that conversion to surgical treatment may be warranted after a period of observational management, if high signal intensity changes are observed on 
T2-weighted MRI. On the other hand, a high signal intensity change on T2-weighted MRI was not associated with the neurological prognosis in patients who required conversion to surgery, similar to some previous studies. ${ }^{16,17}$

There were several limitations to the present study that should be kept in mind when interpreting the results. First, the treatment protocol, including the observational follow-up period, indications for surgical treatment and the type of surgery were not unified in the patients, because this was a retrospective study. Second, there was a possibility that the study was underpowered to identify any significant difference regarding the neurological prognosis, because the sample size in this study was relatively small. Third, although we demonstrated the possibility that spinal cord involvement in CSA was associated with its prognosis, we could not prove this using more detailed methods, such as electrophysiological or pathological methods or recently reported modalities, such as MRI/(18)F-FDG PET fusion imaging. ${ }^{18}$ Although further study will be needed to identify additional prognostic factors for CSA, this study is considered to be a precious study of CSA, because it clearly shows that the signs of spinal cord involvement, including the MRI findings and pyramidal tract sign, can be used as prognostic factors for CSA.

In conclusion, we speculated that the presence of a high signal intensity change on T2-weighted MRI and the pyramidal tract sign reflecting the spinal cord pathology can be used as prognostic factors for patients with CSA, including when the diagnostic criteria for CSA include the patients with the pyramidal tract sign.

\section{DATA ARCHIVING}

There were no data to deposit.

\section{CONFLICT OF INTEREST}

The authors declare no conflict of interest.

\section{DISCLAIMER}

This article has not been published or submitted for publication elsewhere. No benefits in any form have been received or will be received from any commercial party related directly or indirectly to the subject of this article.
1 Brain WR, Northfield D, Wilkinson M. The neurological manifestations of cervical spondylosis. Brain 1952; 75: 187-225.

2 Keegan JJ. The cause of dissociated motor loss in the upper extremity with cervical spondylosis, a case report. J Neurosurg 1965; 23: 528-536.

3 Sobue I, Kato H, Yanagi T. Clinical characteristics of cervical spondylotic amyotrophy. Rinsho Seikeigeka 1975; 10: 999-1006.

4 Yanagi T, Kato $H$, Sobue T. Clinical characteristics of cervical spondylotic amyotrophy. Rinsho Seikeigaku 1976; 16: 520-528.

5 Gebere-Michael SG, Johnston JC, Metaferia GZ, Wuhib MZ, Fernandez HH. Bilaterally symmetric cervical spondylotic amyotrophy: a novel presentation and review of the literature. J Neurol Sci 2010; 290: 142-145.

6 Ito T, Tsuji H, Tamaki T, Miyasaka H, Toyota A. The clinical consideration of the dissociated motor loss syndrome (Keegan) in diseases of the cervical spine. Nippon Seikeigeka Gakkai Zasshi 1980; 54: 131-151.

7 Kameyama T, Ando T, yanagi T, Yasui K, Sobue G. Cervical spondylotic amyotrophy: magnetic resonance imaging demonstration of intrinsic cord pathology. Spine (Phila Pa 1976) 1988; 23: 448-452.

8 Kaneko K, Taguchi T, Toyama K, Kato Y, Azuma Y, Kawai S. Distal-type cervical spondylotic amyotrophy: assessment of pathophysiology from radiological findings on magnetic resonance imaging and epidurally recorded spinal cord responces. Spine 2004; 29: E185-E188.

9 Hattori S, Kawai S. Diagnosis of cervical spondylosis. Orthopaedic Mook 1979; 6: 1340. (Jpn).

10 Jiang SD, Jiang LS, Dai LY. Cervical spondylotic amyotrophy. Eur Spine J 2011; 20: 351-357.

11 Inui Y, Miyamoto H, Sumi M, Uno K. Clinical outcomes and predictive factors relating to prognosis of conservative and surgical treatment for cervical spondylotic amyotrophy. Spine (Phila Pa 1976) 2011; 36: 794-799.

12 Chen CJ, Lyu RK, Lee ST, Wong YC, Wang LJ. Intramedullary high signal intensity on T2-weighted MR images in cervical spondylotic myelopathy: prediction of prognosis with type of intensity. Radiology 2001; 221: 789-794.

13 Wada E, Yonenobu K, Suzuki S, Kanazawa A, Ochi T. Can intramedullary signal change on magnetic resonance imaging predict surgical outcome in cervical spondylotic myelopathy? Spine (Phila Pa 1976) 1999; 24: 455-461 (discussion 462).

14 Yagi M, Ninomiya K, Kihara M, Horiuchi Y. Long-term surgical outcome and risk factors in patients with cervical myelopathy and a change in signal intensity of intramedullary spinal cord on magnetic resonance imaging. J Neurosurg Spine 2010; 12: 59-65.

15 Yukawa Y, Kato F, Yoshihara H, Yanase M, Ito K. MR T2 image classification in cervical compression myelopathy. Spine (Phila Pa 1976) 2007; 32: 1675-1678.

16 Tauchi R, Imagama S, Inoh H, Yukawa Y, Kanemura T, Sato K et al. Risk factors for a outcome following surgical treatment of cervical spondylotic amyotrophy: a multicenter study. Eur Spine J 2013; 22: 156-161.

17 Uchida K, Nakajima H, Yayama T, Sato R, Kobayashi S, Kokubo Y et al. Anterior and posterior decompressive surgery for progressive amyotrophy associated with cervical spondylosis: a retrospective study of 51 patients. J Neurosurg Spine 2009; 11 : 330-337.

18 Uchida K, Nakajima H, Okazawa H, Kimura H, Kudo T, Watanabe S et al. Clinical significance of MRI/(18)F-PET fusion imaging of the spinal cord in patients with cervical compressive myelopathy. Eur J Med Mol Imaging 2012; 39: 1528-1537. 